\title{
A Damage Identification Method based on Differential Gradient of Normalized Strain
}

\author{
Lu Lingling ${ }^{1,}$, Huang Chenguang ${ }^{1, b}$, Wang $\mathrm{Xi}^{1, \mathrm{c}}$ \\ Key Laboratory of Hydrodynamics and Ocean Engineering \\ Institute of Mechanics Chinese Academy of Sciences Beijing, China

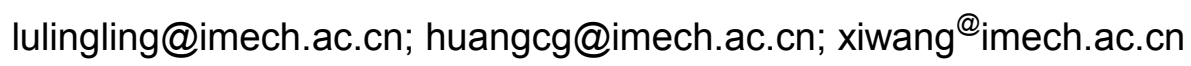

\begin{abstract}
Keywords: Structural Health Monitoring (SHM), Damage Identification, Differential Gradient of Normalized Strain (DGNS), Differential of Strain Contour Density (DSCD), Digital Image Correlation (DIC)
\end{abstract}

\begin{abstract}
A damage identification method based on differential gradient of normalized strain (DGNS) is presented to overcome the disadvantages of traditional static damage identification, such as the complicacy of measurement system and the limited measurement points etc. Two numerical simulations were conducted on a dog-bone specimen to verify the feasibility of the method. In the experiment, differential of strain contour density (DSCD), which has the same physical meaning with DGNS, significantly improves the smoothness and visualization of field information. Both the simulation and experiment results show that, DGNS (DSCD) is capable of describing the structural damage property meanwhile effectively isolates the damaged areas from regions with inhomogeneous deformation due to geometric inhomogeneity. Moreover, DGNS (DSCD) is a structural intrinsic parameter, and independent on external loads.
\end{abstract}

\section{Introduction}

As a result of collisions with foreign objects, fatigue loading and corrosive environments et al., critical structural systems such as aircraft, spacecraft, bridges, and offshore platforms continuously accumulate damage in their service environments. Damage, which is considered as an index for the differential of the mechanical properties of structures or material, usually consists of many forms, such as plastic deformation, cracks, micro-holes and gaps. For such structures, periodic inspections and maintenance are mandatory to ensure safety [1]. Non-destructive detection (NDD) method to detect damage status of engineering structures has become an important issue [2]. However, most of NDD methods including static identification and dynamic identification on the contact measurement could not be fully used in some special situations, such as high temperature and corrosive environments.

Digital image correlation (DIC), proposed by Peters [3] and Yamaguchi [4] in 1982, as an optical measurement method, has been widely used for characterization of full-field deformation and its evolutions under various conditions, due to its characteristics of non-contact and simple measuring requirements. It has been used to get the displacement and strain distribution of the target structure for analyzing the crack growth [5 7], necking during monotonic loading, phase transformation in materials and so on.

There are little reports on the application of the method combining DIC with SHM. The reason could be in SHM system intrinsic parameters of structures, such as frequency [8 9], modal shape, modal strain energy, flexibility [10] and stiffness information, are usually used for detecting and evaluating damage. Nevertheless, parameters obtained by DIC are usually dependent on both the structural configuration and external loads.

In this work, a damage identification method based on the differential gradient of normalized strain (DGNS) is proposed. On the one hand, the proposed method is a non-contact technology, suitable for more situations than traditional contact measurement. On the other hand, the damage index DGNS is a structural intrinsic parameter, which can identify the damage like plastic deformation and cracks, and isolate the damaged areas from the strain concentration areas due to structural geometric configurations effectively. 


\section{The principle of the method}

As damage occurs in the materials or structures, the distribution of stress around the damaged region would change, causing the inhomogeneity of strain. The geometrically inhomogeneous features in the structure such as holes and grooves also cause the heterogeneity of deformation. If the strain is used as the damage index, it is difficult to detect the real damage region in the early damage stage since the heterogeneity caused by the structure geometry may covers up that in damage regions. So the damage can only be identified in the later stage when the structure is severely damaged.

In consequence, a reasonable damage index should be proposed to distinguish the real damage and the geometric inhomogeneity such that we can identify the real damage regions effectively in the early damage stage. Differential of gradient normalized strain (DGNS) is proposed, used as the damage index in the paper, of which the feasibility is proved as follows.

The relation between the strain of a structure and the external load can be written as:

$$
\varepsilon_{N D}=\alpha_{N D} * f
$$

where $N$ is the node number, $D$ is the direction of strain, $f$ is the load and $\alpha_{N D}$ is a parameter related to the geometry, the material properties and the deformation stages of the structure. Then, the normalized strain $\rho_{N D}$ is defined as:

$$
\rho_{N D}=\frac{\varepsilon_{N D}-\varepsilon_{M I N D}}{\varepsilon_{M A X D}-\varepsilon_{M I N D}}
$$

where $\varepsilon_{M A X D}$ and $\varepsilon_{M I N D}$ are the maximal and minimum value in the strain field respectively. Substituting (1) into (2), we can obtain:

$$
\rho_{N D}=\frac{\alpha_{N D}-\alpha_{M I N D}}{\alpha_{M A X D}-\alpha_{M I N D}}
$$

We can see $\rho_{N D}$ is independent on $f$ and only related to $\alpha_{N D}$. The gradient of normalized strain (GNS) $\xi_{N D}$ is defined as:

$$
\xi_{N D}=\frac{\rho_{(N+1) D}-\rho_{(N-1) D}}{x_{(N+1) D}-x_{(N-1) D}}
$$

where $x_{(N+1) D}$ and $x_{(N-1) D}$ are the coordinates of the nodes next to the node $N$. Substituting (3) into (4), we can obtain (5). From (5), we can see that GNS only depends on parameter $\alpha_{N D}$ which only related to the geometry and material property of the structure.

$$
\xi_{N D}=\frac{\alpha_{(N+1) D}-\alpha_{(N-1) D}}{\left(x_{(N+1) D}-x_{(N-1) D}\right) *\left(\alpha_{M A X D}-\alpha_{M I N D}\right)}
$$

\section{Numerical simulations}

In this section, two numerical simulations are conducted to validate the proposed method. The first case is a dog-bone structure with a groove on one side. The groove is to represent the geometric inhomogeneity. The damage considered in this case is plastic deformation. The other case is a dog-bone structure with a square-shape hole and a crack. The hole is to represent the geometric inhomogeneity while the crack is the damage to identify. ANSYS is used to calculate the deformation of the structure. The GNS and DGNS are computed with a developed MATLAB program. 


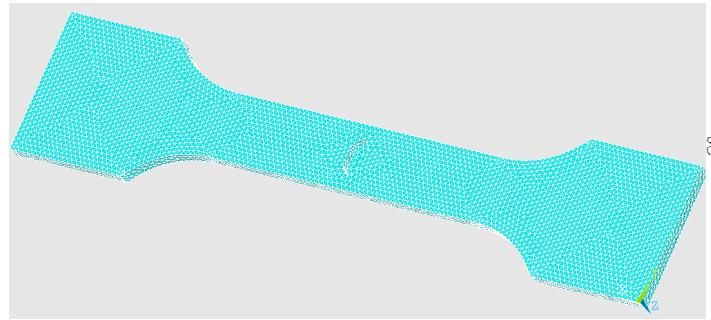

(a)

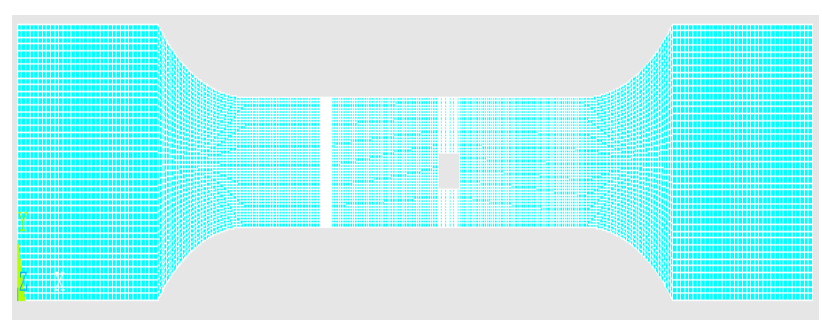

(c)

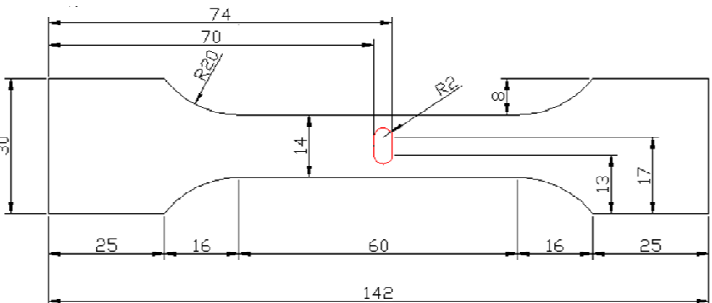

(b)

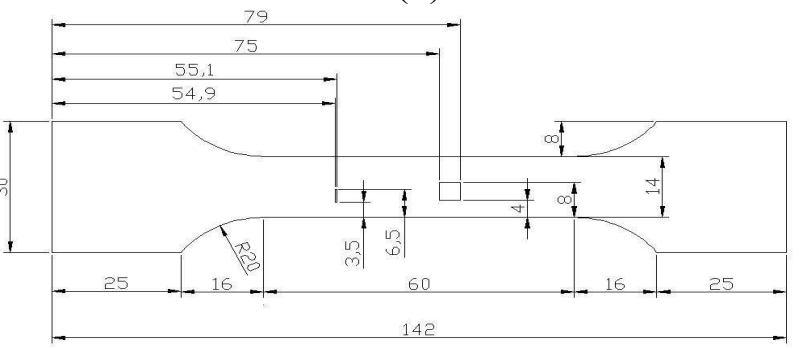

(d)

Fig. 1. The information of the specimens: (a),(b) is the finite element model and dimension of case I respectively; (c) and (d) is that of case II (Unit: $\mathrm{mm}$ )

\section{Case I.}

The structure is a dog-bone specimen with the dimension of $142 \times 20 \times 3 \mathrm{~mm}$ as shown in Fig. 1(b). A groove with a depth of $1 \mathrm{~mm}$ is located at the center of the structure. The material (aluminium alloy) is considered to be elastic/plastic with linear hardening (Young's modulus, $E=73 \mathrm{GPa}$, yield strength, $\sigma_{\mathrm{s}}=325 \mathrm{Mpa}$, plastic modulus, $\mathrm{E}_{\mathrm{p}}=1.5 \mathrm{Gpa}$, Poisson ratio of $\mu=0.34$, and mass density of $\left.\rho=2780 \mathrm{~kg} / \mathrm{m}^{3}\right)$. The tension loads were applied to nodes on the right side of the specimen in a displacement mode. There are totally 4 simulations A, B, C and D (A, B: elastic stage; C, D: plastic stage), with the right-side displacement as $7.1 \mu \mathrm{m}, 56.8 \mu \mathrm{m}, 284 \mu \mathrm{m}$ and $360 \mu \mathrm{m}$ respectively. The deformation field on the surface of the structure opposite the grooved one is used to detect the damage.

The total mechanical strain, plastic strain and GNS field information of the specimen for 4 simulations are shown in Fig. 2. Taking case A as a reference, the DGNS fields, e. $g$ the difference of GNS field with the reference case are shown in Fig. 3. From Fig. 2, we can see the strain filed of the 4 cases is quite similar whether there is plastic deformation or not. The DGNS filed is uniform as shown in the top figure of Fig. 3 when there is no plastic deformation, while the groove area is highlighted once plastic deformation occurs. The amplitude of the DGNS filed increase as the plastic deformation amplifies. Comparing the plastic strain field in Fig.2 and the DGNS field in Fig.3, we can see that DGNS field clearly match the region where plastic deformation occurs. In other words, DGNS can effectively indentify plastic deformation as a damage index.

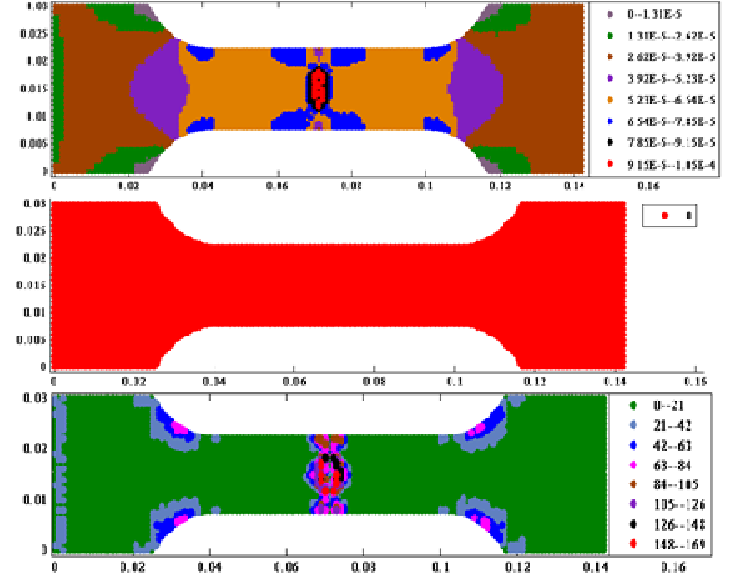

(a)

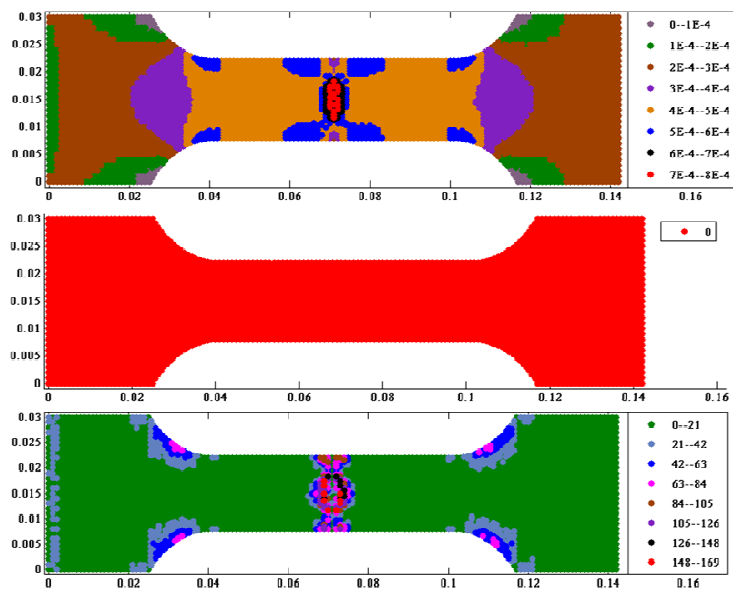

(b) 


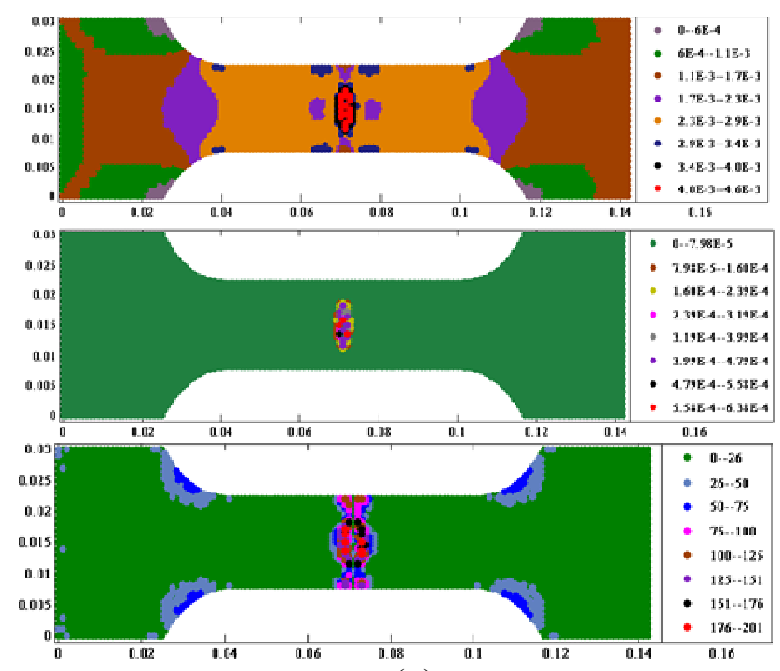

(c)

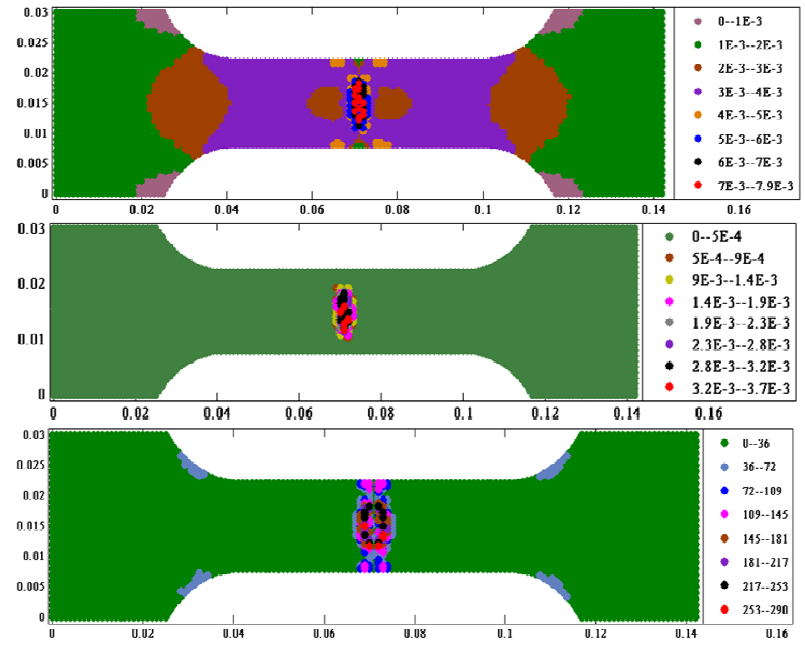

(d)

Fig. 2. The results of the structure at (a) simulation A; (b) simulation B; (c) simulation C; (d) simulation D; (Top: the total mechanical strain field; middle: the plastic strain field; bottom: the GNS field)

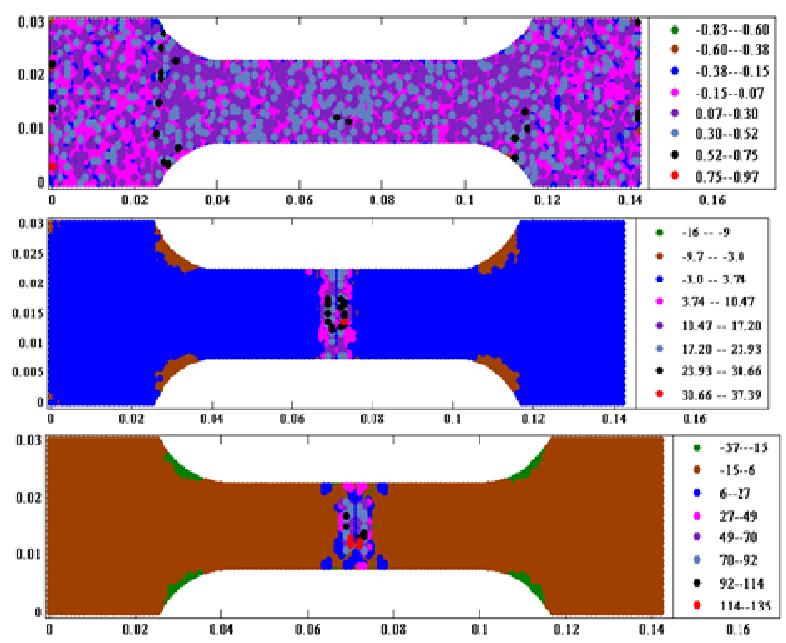

Fig. 3. The DGNS field of simulation B (top), C (middle), and D (bottom) with simulation A as a reference

\section{Case II.}

The structure (damaged structure) is a dog-bone structure with a square-shape hole and a crack. The hole is to represent the geometric inhomogeneity while the crack is the damage to identify. A structure having a hole but without the crack (intact structure) is considered as a reference. The geometry of the structure is shown in Fig. 1(d). The material is the same as Case I. The tension loads were applied to nodes on the right side of the specimen in a displacement mode. There are totally 5 simulations (A, B: elastic stage; C, D, E: plastic stage), with the displacement $7.1 \mu \mathrm{m}, 42.6 \mu \mathrm{m}$, $113.6 \mu \mathrm{m}, 170 \mu \mathrm{m}$ and $284 \mu \mathrm{m}$ respectively.

The simulation results are shown in Fig. 4 9. The GNS, displacement and strain distribution fields of the 5 simulations are similar, only different in amplitudes so only the simulation E's three fields information are shown in Fig. 4, Fig. 6, Fig. 8 (x axis: the extruding direction, unit: mm). Fig. 5, Fig. 7 and Fig. 9 show the relationship between the differential of displacement (DOD), the differential of strain (DOS), DGNS and the external loads respectively. 


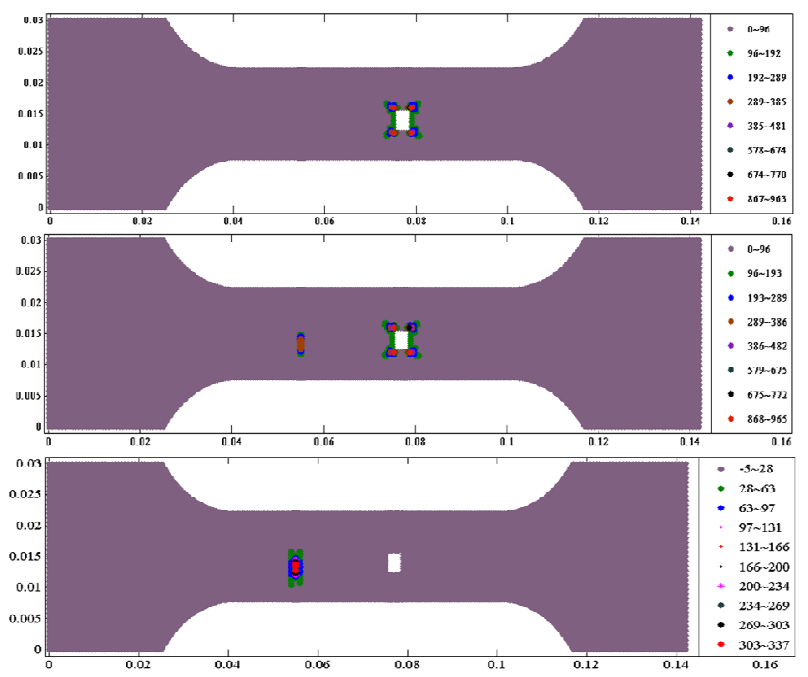

Fig. 4. The GNS field: intact specimen (top); damaged specimen (middle); the differential information (bottom)

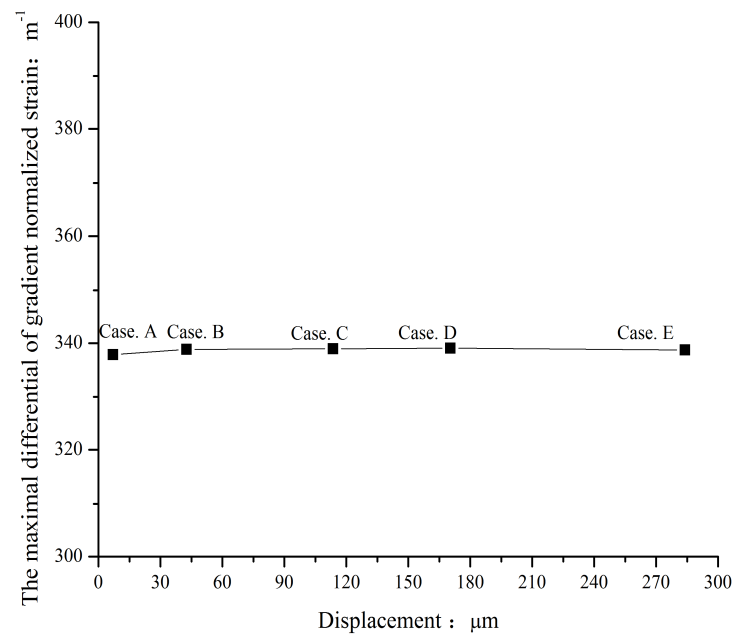

Fig. 5. The displacement $\sim$ maximal DGNS curve

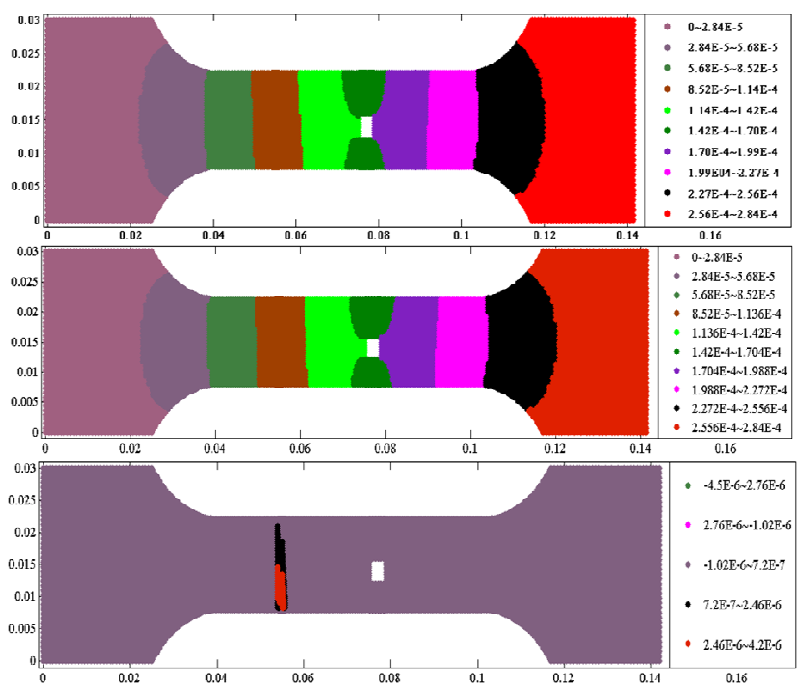

Fig. 6. The displacement fields: intact specimen (top); damaged specimen (middle); the differential information (bottom) 


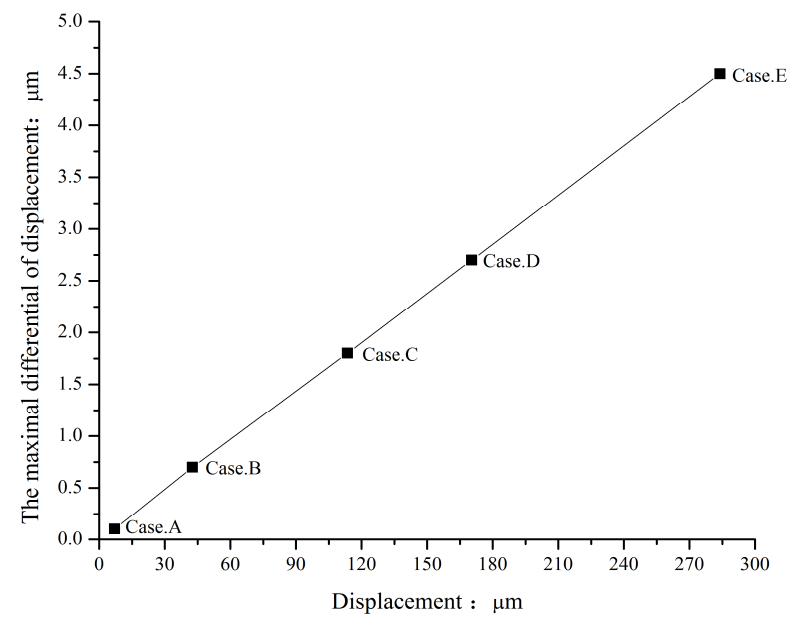

Fig. 7. The displacement $\sim$ maximal differential of displacement curve
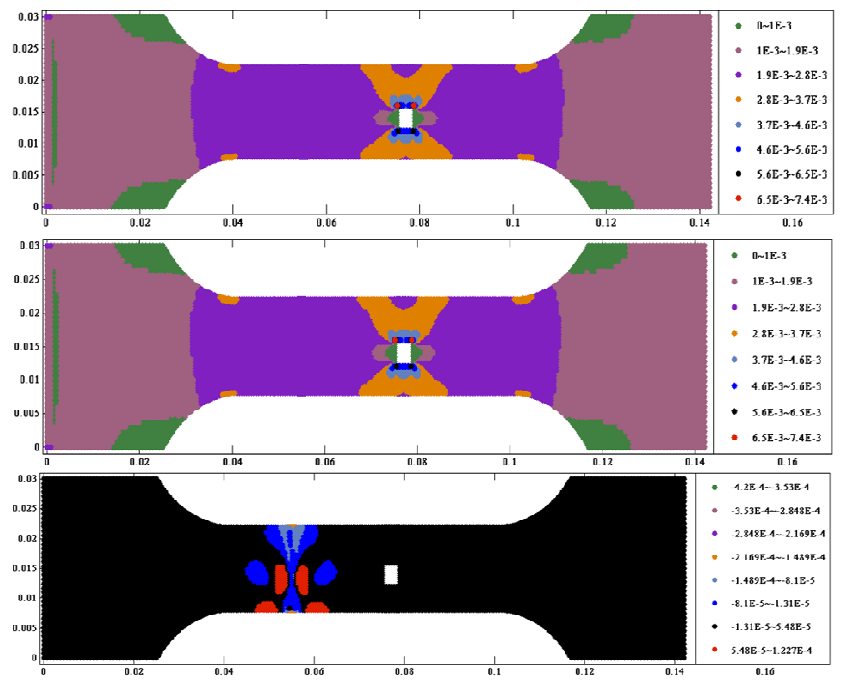

Fig. 8. The strain fields: intact specimen (top); damaged specimen (middle); the differential information (bottom)

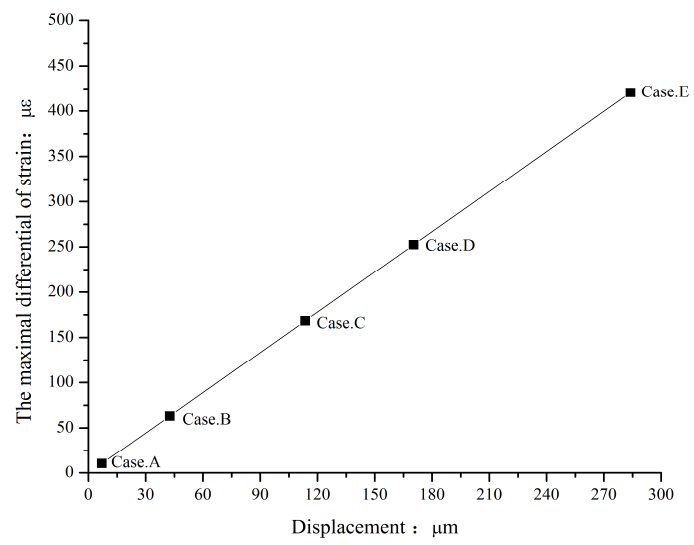

Fig. 9. The displacement $\sim$ maximal differential of strain curve

The GNS field can clearly illustrate the localized deformation around the hole or crack as shown in Fig. 4 in both damaged and intact structures. In the DGNS field, only the crack is highlighted, indicating the DGNS is an effective damage index which can isolate the damage (crack in this case) from the geometric inhomogeneity (hole in this case). As the load increases, the maximum of DGNS stays a constant, illustrating DGNS is an intrinsic feature of structures and independent from external loads. The results show that DGNS is a reasonable effective index for damage identification. 
From the displacement and strain fields of the intact and damaged specimen (as shown in Fig.6 and Fig. 8), we can see the deformation fields around the hole is so severe that it conceal the existence of crack. Even though the differential fields with the intact structure (DOD and DOS) can highlight the crack as shown in the bottom figure of Fig.6 and Fig.8, the effectiveness of using them as an index for damage identification is limited by their relatively small values. For example, in simulation $\mathrm{C}$, the maximum DOD and DOS are only $1.8 \mu \mathrm{m}$ and $175 \mu \varepsilon$ respectively, which are difficult to be captured according to the present accuracy of DIC technology. Therefore it is difficult to identify the damage region at small loads by the DOD or DOS in the early damage stage.

\section{Experiments}

Experiment setup. A tensile test on the dog-bone specimen with the groove $1 \mathrm{~mm}$ depth as shown in Fig.1 (b) was carried out to verify DGNS is a sensitive index for the plastic deformation. The test was conducted on the WDW-50E test machine with a loading rate of $0.2 \mathrm{~mm} / \mathrm{min}$ at room temperature. As shown in Fig. 10(a), the specimen surface was painted with a speckle pattern and images of the surface were captured by a CCD camera. The image size is $1600 \times 500$ pixels and the image acquisition rate was 2 fps (frame per second).

DIC technique gives the displacement field of the deformation from the correlation of a series of images. DGNS, which is the $2^{\text {nd }}$ derivative of the displacement field, is affected significantly by the noise during DIC measurements. Here we proposed an alternative parameter to DGNS to overcome this issue in practice, which is the differential of strain contour density (DSCD) and defined as:

$$
\theta_{N D}=\frac{\varepsilon_{\max D}-\varepsilon_{\min D}}{\varepsilon_{M A X D}-\varepsilon_{M I N D}} \times M
$$

where, $\theta_{N D}$ is the strain contour density (SCD) of node $N ; \varepsilon_{\max D}, \varepsilon_{\min D}, \varepsilon_{M A X D}, \varepsilon_{M I N D}$ are local and global maximum and minimum strains as shown in Fig. 11; $M$ is the number of contour lines. $\mathrm{R}$ is the size of the region under examination. DSCD is intrinsically the same as DGNS since it also represents the gradient of the strain field but in a more practical way. The DSCD is proved to be approximately independent on $\mathrm{R}$ when $\mathrm{R}$ is between $3 \mathrm{~L}$ and $10 \mathrm{~L}$ ( $\mathrm{L}$ : the element size). In the analysis of experimental results, we chose $\mathrm{M}=30$, and $\mathrm{L}=7$ pixels and $\mathrm{R}=4 \mathrm{~L}$.

The displacement and strain field of the structure were obtained by a DIC code provided by professor Shaopeng Ma of Beijing institute of technology. Then the SCD and DSCD field were computed by a developed MATLAB program as (6).

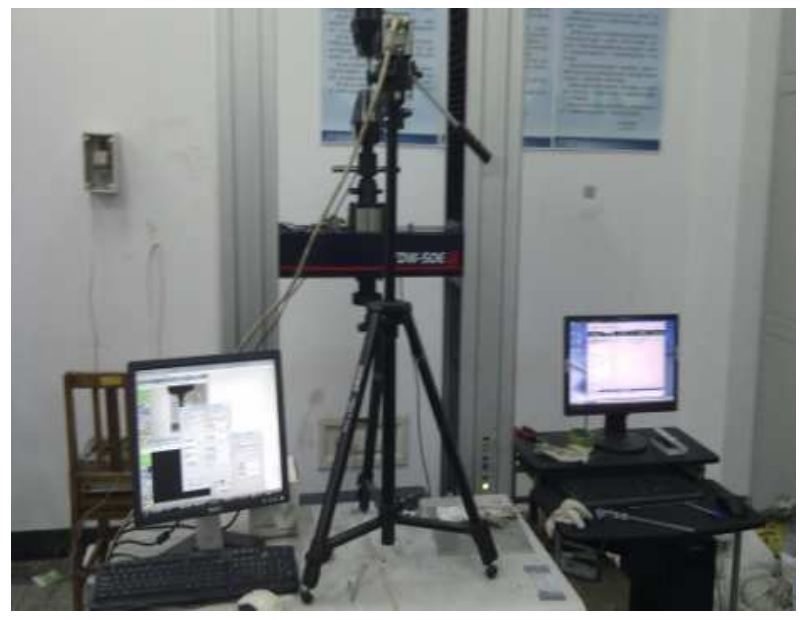

(a) 


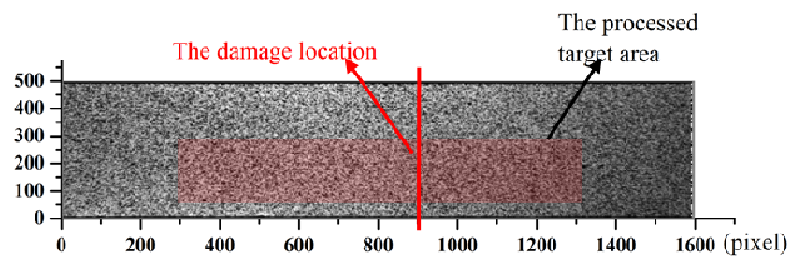

(b)

Fig. 10. Schematic of the experiment: (a) the experimental set-up; (b) introduction of the processed area

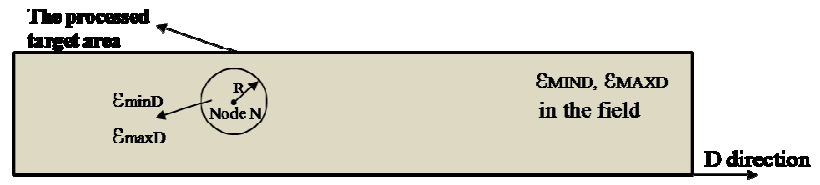

Fig. 11. Introduction of the 4 parameters in (6)

\section{Results}

The load $\sim$ strain curve of the dog-bone specimen is shown in Fig. 12. The SCD fields are shown in Fig. 13. Taking the result corresponding to point A on the load $\sim$ strain curve as a reference, the DSCD fields, i.e. the difference of strain contour density field with the reference case, are shown in Fig.14. The horizontal and vertical coordinates are pixels, and the value in the caption represents the differential number of contour line compared with the reference.

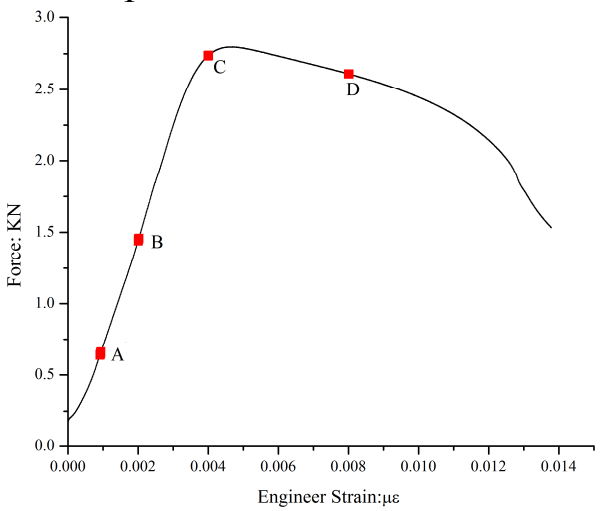

Fig. 12. The strain $\sim$ force curve of the specimen
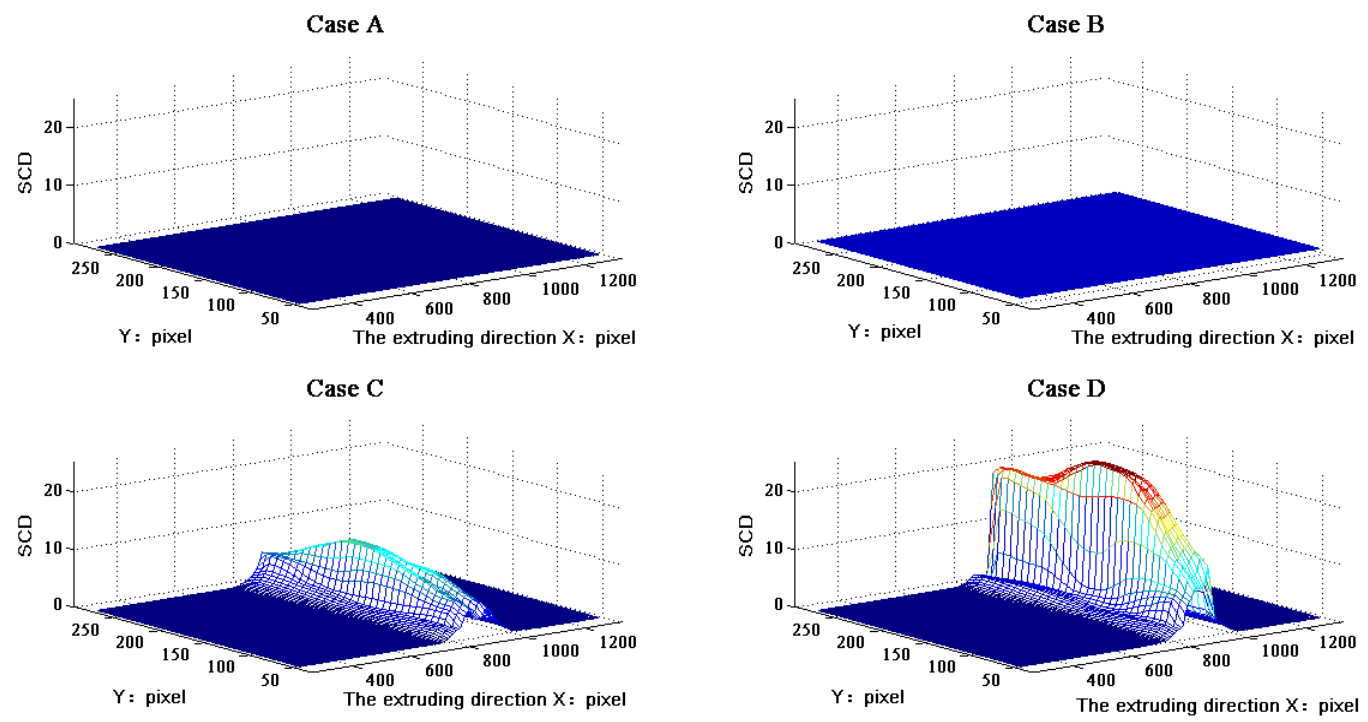

Fig. 13. The SCD fields information 

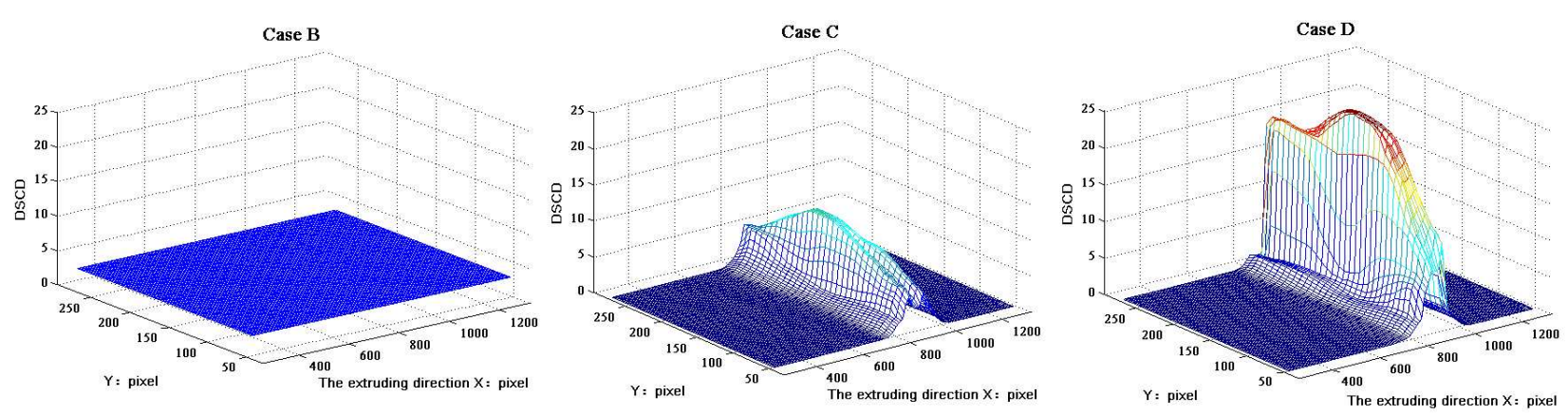

Fig. 14. The DGNS field

From Fig. 13, the SCD field is uniform in the early elastic stage (point A and B on the load $\sim$ strain curve). However, in the plastic stage (point $\mathrm{C}$ and $\mathrm{D}$ ), regions around the groove firstly enter into plastic stage and display the inhomogeneity of deformation, as shown in SCD fields. From the DSCD field in Fig.14, we can see that DSCD field clearly match the region where plastic deformation occurs, proving DSCD can effectively indentify plastic deformation as a damage index. The experiment results were also consistent with the simulations in Case I.

Obviously, the accuracy of the damage identification method proposed in this paper is restricted by the precision of strain identification of DIC technology. More accurate the DIC technology is, more effective the method presented would be. There are lots of studies going on to improve the accuracy of DIC ${ }^{[116]}$. Currently, the displacement resolution can reach about $0.005 \sim 0.1$ pixel, and the strain resolution is floating around hundreds of $\mu \varepsilon$. Therefore, the proposed method is strongly related to the advances of the DIC technique.

\section{Conclusion}

The conclusions obtained from the numerical analysis and the experiments are as follows:

(1) For the static damage identification, a method based on DGNS is proposed in the paper, which uses the gradient of the strain field as the damage index to monitor the health of the structure.

(2)From the numerical simulation results, we can see that DGNS is capable of identifying the structural damage property like plastic deformation and cracks, meanwhile it can effectively isolate the damaged areas from the heterogeneity of deformation regions caused by geometric inhomogeneity. Moreover, DGNS could identify the damage in early stage as it has been proved a structural intrinsic parameter.

(3)Experiment results indicate that in practice DSCD instead of DGNS can effectively identify plastic deformation as proved in Section 3.1. The damage identification ability of the proposed method is limited by the precision of DIC technology.

\section{References}

[1] J. Chen, J. A. Garba. On-Orbit damage assessment for large space structures. AIAA Journal, Vol.26, No.9, 1988, pp. 1119-1126

[2] Y.J. Yan, L. Cheng, Z.Y. Wu, L.H. Yam. Development in vibration-based structural damage detection technique. Mechanical systems and signal processing, Vol.21, 2007,pp. 2198-2211

[3] Peters W H, Ranson W F. Digtial imaging techniques in experimental stress analysis. Optical Engineering, Vol.21, 1982, pp. 427-431

[4] Yamaguchi I. Simplified laser-speckle strain gauge. Optical Engineering, Vol.21, 1982, pp. 436-440

[5] T.Niendorf, J. Dadda, D. Canadinc, H. J. Maier, I. Karaman. Monitoring the fatigue-induced damage evolution in ultrafine-grained interstitial-free steel utilizing digital iamge correlation. Materials science and engineering A, Vol.517, 2009, pp. 225-234 
[6] Marion Risber, Pierre Feissel, Thierry Roland, Delphine Brancherie, Jean-Marc Roelandt. Digital image correlation technique: application to early fatigue damage detection in stainless steel. Procedia engineering, Vol.2, 2010, pp. 2219 2227

[7] M. Li, J. Zhang, C. Y .Xiong, J. Fang, J. M. Li, Y. Hao. Damage and fracture prediction of plastic-bonded explosive by digital image correlation processing. Optics and lasters engineering, Vol. 43, 2005, pp. 856-868

[8] Sophia Hassiotis, Garrett D. Jeong. Identification of stiffness reductions using natural frequcies. Journal of engineering mechanics, Vol.10, 1995, pp. 1106-1113

[9] V. Ramamurti, Sumanta Neogy. Effect of cracks on the natural frequency of cantilevered plates-A Rayleigh-Ritz solution. Mechanics of structures and machines, Vol.26, 1998, pp. 131-143

[10]Pandey A. K, Biswas M. Experimental verification of flexibility difference method for locating damage in structures. Journal of sound and vibration, Vol.184, No. 2, 1995, pp. 311-328

[11]Pan Bing, Xu Boqin, Chen Ding, et al. Sub-pixel registration using quadratic surface fitting in digital image correlation. Acta Metrologica Sinca,Vol.26, No.2, 2005, pp. 128-134

[12] Yang Yong, Wang Yanlei, Li Ming, et al. Research of high-accuracy digital image correlation measurement system. Acta Optica Sinica, Vol.26, No.2, 2006, pp.197-201

[13] Yu Qifeng. Image based precise measurement and motion measurement. Beijing: Science Press, 2002

[14]Bruck HA, McNeil SR, Sutton MA, et al. Digital image correlation using Newton-Rapshon method of partial differential correction. Experimental Mechanics, Vol.29, No.3, 1989, pp. 261-267

[15]Pilch A, Mahajan A, Chu T. Measurement of whole-field surface displacements and strain using a genetic algorithm based intelligent image correlation method. Journal of dynamic Systems, Measurement and Control. Transactions of the ASME, Vol.126, No.3, 2004, pp. 479-488

[16]Pan Bing, Xie Huimin, Dai Fulong. An investigation of sub-pixel displacement registration algorithms in digital image correlation. Chinese Journal of Theoretical and applied mechanics, Vol.39, No.2, 2007,pp.24 